\title{
Sufficiency of unidirectional allostery in KaiC in generating the cyanobacterial circadian rhythm
}

\author{
Shin-ichi Koda* and Shinji Saito ${ }^{\dagger}$ \\ Department of Theoretical and Computational Molecular Science, \\ Institute for Molecular Science, 38 Nishigo-Naka, \\ Myodaiji, Okazaki, Aichi 444-8585, Japan
}

(Dated: April 2, 2020)

\begin{abstract}
The clock protein of cyanobacteria KaiC forms a homohexamer with two ring-shaped domains, $\mathrm{C} 1$ and $\mathrm{C} 2$. These domains undergo several domain-specific conformational transitions and allosterically communicate to generate a circadian rhythm. Interestingly, experiments show a possibility that $\mathrm{C} 2$ is independent of $\mathrm{C} 1$. However, detailed interplay among them remains elusive. Here we propose a mathematical model, which explicitly considers the interplay. The allostery in KaiC is here modeled to be unidirectional from $\mathrm{C} 2$ to $\mathrm{C} 1$. We demonstrate that the unidirectional allostery is sufficient for the circadian rhythm by showing the quantitative reproducibility of various experimental data, including temperature dependence of both phosphorylation oscillation and ATPase activity. Based on the present model, we further discuss possible functional roles of the unidirectional allostery particularly in the period robustness against both protein concentration and temperature.
\end{abstract}

\footnotetext{
*koda@ims.ac.jp

$\dagger$ shinji@ims.ac.jp
} 


\section{INTRODUCTION}

Circadian clocks are biological timing systems embedded in most living organisms and enable the organisms to anticipate daily changes in the environment and to adjust their biological activities. The simplest circadian clock is that of cyanobacteria, where the core oscillator is composed of only three proteins, KaiA, KaiB, and KaiC[1]. Interestingly, this circadian rhythm can be reconstituted in a test tube just by mixing the three proteins with ATP, which results in a nearly 24-hour periodic oscillation of phosphorylation of KaiC[2]. In addition to this self-sustaining oscillation, the KaiABC oscillator possesses several fundamental functions as a biological clock. For example, the period of the oscillator is robust against environmental perturbations such as temperature[2] and concentrations of the proteins[3]. The oscillator is further entrained by various periodic environmental changes, including temperature[4] and ATP/ADP ratio in buffer[5]. This simple yet functional system has thus attracted considerable interests in elucidating the molecular origins of circadian rhythm.

KaiC is the central component in the KaiABC oscillator, and in the presence of ATP, forms a homohexamer with two ring-shaped domains, C1 and C2[6, 7]. Both C1 and C2 have ATPase activities. C2 also has autokinase and autophosphatase activities, where two residues near the ATP binding site in C2, Ser431 and Thr432, are phosphorylated and dephosphorylated. With the help of KaiA and KaiB, (de)phosphorylation occurs in the following order: $\mathrm{ST} \rightarrow \mathrm{SpT} \rightarrow \mathrm{pSpT} \rightarrow \mathrm{pST} \rightarrow \mathrm{ST}[8,9]$, where $\mathrm{S} / \mathrm{pS}$ and $\mathrm{T} / \mathrm{pT}$ are the unphosphorylated/phosphorylated states of Ser431 and Thr432, respectively.

KaiA promotes phosphorylation of $\mathrm{KaiC}[10,11]$ by acting on the C-terminal tail of $\mathrm{C} 2[12-$ 15]. It facilitates the exchange of bound ADP with exogenous ATP[16], suggesting that the enhanced ADP/ATP exchange supplies abundant phosphate for phosphorylation reactions in the form of ATP, and shifts the equilibrium of phosphorylation reactions toward phosphorylated states.

KaiB, on the other hand, switches the oscillation phase to dephosphorylation by inhibiting KaiA activity[17, 18]. When $\mathrm{C} 2$ is adequately phosphorylated, KaiB binds to $\mathrm{C} 1$ and strongly sequesters KaiA from C2 by forming C1 - KaiB - KaiA complex[19]. Indeed, this complex has recently been observed experimentally[20, 21]. After this sequestration, KaiA no longer promotes phosphorylation, and C2 starts to dephosphorylate, thus the phosphorylation oscillation occurs. 
During the oscillation, C1 and C2 allosterically communicate and regulate the (dis)assembly of KaiA and KaiB through several domain-specific conformational transitions. For example, recent studies on the KaiABC complex[20, 21] have revealed that KaiB can bind to an ADP-bound conformation of $\mathrm{C} 1[22]$. On the other hand, $\mathrm{C} 2$ has the buried and exposed states of the C-terminal tail[14, 15], and KaiA can bind only in the latter state. C2 also takes the rigid and flexible conformational states that regulate C1-C2 stacking[19, 23]. Yet, it remains elusive how these multiple conformational transitions interplay each other and link the chemical reactions of ligands, e.g. ATP hydrolysis and (de)phosphorylation reaction, with the (dis)assembly of Kai proteins. In particular, we here focus on an unaccounted experimental result that modulations on $\mathrm{C} 1$ do not show apparent effect on the dephosphorylation process in C2[24, 25]. Interestingly, this C1-independent C2 dephosphorylation contradicts an intuition that $\mathrm{C} 1$ and $\mathrm{C} 2$ should bidirectionally interact to generate the oscillation.

In this article, we conduct a quantitative investigation with a novel mathematical reaction model to clarify the relation between the functions of the KaiABC oscillator and the interplay among the domain-specific conformational transitions. Our model has four main features below. First, the model explicitly considers multiple domain-specific conformational transitions and the interplay among them. In particular, we hypothetically design the allosteric communication in KaiC to be unidirectional from $\mathrm{C} 2$ to $\mathrm{C} 1$ so that the model can realize the $\mathrm{C} 1$-independent $\mathrm{C} 2$ phosphorylation mentioned above. In contrast, previous models considering conformational states[26-29] have assumed only a single global conformational transition of overall KaiC. This single global conformational transition, where C1 and C2 bidirectionally interplay, could be an oversimplified assumption because further assumptions are needed to realize the C1-independent C2 dephosphorylation. Second, in contrast to conceptually simplified models, the present model is described as a set of elementary reactions, which consists of the chemical reactions of ligands, (dis)assembly of KaiA and $\mathrm{KaiB}$, and the conformational transitions of KaiC. With this representation, the present model allows detailed investigations of the clock functions on molecular basis. Third, owing to the representation based on elementary reactions, an Arrhenius temperature dependence is introduced to rate or equilibrium constant of each elementary reaction, which enables investigations on the temperature compensation of the period. Lastly, the parameter values of the present model are determined by an automatic optimization, which encourages the 
reproducibility of several fundamental experimental data. This approach reduces arbitrariness in the parameter values compared to the manually determined values, which are often used to explain a specific experimental result. Thus, the present parameter values improve the reliability of the model. To our knowledge, there has been no previous model satisfying all the features above.

With the present model, we investigate the relation between the elementary reactions and clock functions of the KaiABC oscillator. In particular, we focus on the hypothetical assumption of the unidirectional allostery from $\mathrm{C} 2$ to $\mathrm{C} 1$. We first show that the unidirectional allostery is sufficient to generate the circadian rhythm by demonstrating that the present model can indeed reproduce various experimental results, including temperature dependence of both phosphorylation oscillation and ATPase activity. Based on the present model, we further discuss possible functional roles of the unidirectional allostery in the generation of collective oscillation and in the period robustness against both protein concentration and temperature.

\section{RESULTS}

\section{Model setup}

The present model consists of elementary reactions described in this subsection, which is organized as follows. We first describe the processes at each ATP binding site of C1 and C2. Then, we summarize the domain-specific conformational transitions of $\mathrm{C} 1$ and $\mathrm{C} 2$, together with the (dis)assembly of KaiA and KaiB. Lastly, we explain the allosteric communication between $\mathrm{C} 1$ and $\mathrm{C} 2$ in the present model, which is unidirectional from $\mathrm{C} 2$ to $\mathrm{C} 1$. The details of the model are found in Method.

At each C1 ATP binding site in the present model, only ATP hydrolysis and the ADP/ATP exchange are considered. ATP synthesis is neglected as it is supposed to be very slow due to a high energy barrier. The effect of inorganic phosphate produced by ATP hydrolysis is also ignored for simplicity. The ADP/ATP exchange actually consists of two successive processes, i.e. the release of bound ADP and incorporation of exogenous ATP. In the present model, however, these two processes are combined and referred to as the ADP/ATP exchange because the incorporation of ATP is rapid[7]. 
The processes at each C2 ATP binding site in the present model are summarized in Fig. 1. We use the following notations for phosphorylation and bound-nucleotide states: U (unphosphorylated); S or T (either Ser431 or Thr432 is phosphorylated, respectively); and D (doubly phosphorylated), with a subscript T or D to indicate a bound nucleotide, ATP or ADP, respectively. In $\mathrm{C} 2$, we consider ATP hydrolysis and ADP/ATP exchange as in C1. Moreover, we consider phosphorylation and dephosphorylation reactions of Ser431 and Thr432, in which a phosphate is transferred between the bound nucleotide and either of Ser431 and Thr432[30, 31]. Note that the processes in each ATP binding site in KaiC are the same as Paijmans's model[29].

It has been experimentally suggested that the change of the ADP/ATP exchange in $\mathrm{C} 2$ induces an equilibrium shift toward (de)phosphorylated states of $\mathrm{C} 2[16]$. The present model designs the sequential phosphorylation of $\mathrm{C} 2$ as schematically shown in Fig. 1, in which the equilibrium shift is drawn by thick arrows. Assuming that (de)phosphorylation of Thr432 is faster than that of Ser431, an enhanced ADP/ATP exchange promotes the phosphorylation via the $\mathrm{T}$ state (Fig. 1A), whereas a suppressed ADP/ATP exchange results in the dephosphorylation via the $\mathrm{S}$ state (Fig. 1B).

In the present model, one C1-specific conformational transition, between KaiB-bindingcompetent (BC) and -incompetent (BI) conformational states, is considered (Fig. 2A). We assume that KaiB can bind to only C1 in the BC state and that KaiA can bind strongly to the KaiB-C1 complex, which eventually results in the sequestration of KaiA from C2. Moreover, the conformational transition from BI to BC state is assumed to be promoted when $\mathrm{C} 1$ binds abundant ADP rather than ATP, as suggested experimentally[20, 21, 32, 33]. Under this assumption, switching between phosphorylation and dephosphorylation is controlled by the $\mathrm{ADP} / \mathrm{ATP}$ exchange in $\mathrm{C} 1$ because the exchange determines the amount of $\mathrm{ADP}$ in $\mathrm{C} 1$, which is the trigger of the conformational transition from BI to BC state. Specifically, a suppressed ADP/ATP exchange accumulates ADP molecules from ATP hydrolysis and consequently increases the BC state of $\mathrm{C} 1$ and the KaiA-KaiB-C1 complex. This mechanism is consistent with the recent experimental result that the KaiB binding requires the ATP hydrolysis of C1 in advance[34].

In addition, two types of C2-specific conformational transitions are considered (Fig. 2B): one is between the rigid and flexible conformational states of $\mathrm{C} 2[23]$, and the other is between the buried and exposed conformational states of the C-terminal tail[14, 15]. Based on the two 
experimental results showing that the phosphorylation of $\mathrm{C} 2$ rigidifies $\mathrm{C} 2[23]$ and buries the tail[15], we assume that the exposed state of the tail is absent when $\mathrm{C} 2$ is in the rigid state. Hence, only three combinational states, i.e. rigid-buried, flexible-buried, and flexible-exposed states, are considered. Moreover, we assume that KaiA can bind to C2 only when C2 is in the exposed state. To realize the experimental result that KaiA facilitates the ADP/ATP exchange in $\mathrm{C} 2[16]$, we also assume that the ADP/ATP exchange in C2 is allowed only in the exposed state. With these assumptions, the KaiA binding promotes the ADP/ATP exchange through the stabilization of the exposed state, i.e. the equilibrium shift of Fig. 2B to right.

For the allosteric communication between $\mathrm{C} 1$ and $\mathrm{C} 2$ in the present model, we hypothetically assume that $\mathrm{C} 2$ in the rigid conformational state prohibits the ADP/ATP exchange in $\mathrm{C} 1$. This idea originates from our assumption that the $\mathrm{C} 1-\mathrm{C} 2$ stacking, which concurrently occurs with the rigidification of $\mathrm{C} 2[19,23]$, prohibits the ADP/ATP exchange. In the present model, however, we do not explicitly describe the C1-C2 stacking for simplicity. We here emphasize that this allostery is unidirectional, i.e. the status of $\mathrm{C} 1$ does not affect C2. Thus, the C1-independent $\mathrm{C} 2$ dephosphorylation $[19,23]$ is realized without further assumptions in the present model.

Figure 2D illustrates the overview of the entire oscillation process in the present model explained above. Starting from KaiA-C2 binding (left figure of Fig. 2D), which stabilizes the exposed conformational state of $\mathrm{C} 2$ and promotes the ADP/ATP exchange and phosphorylation of $\mathrm{C} 2$, phosphorylated $\mathrm{C} 2$ rigidifies itself and induces $\mathrm{C} 1-\mathrm{C} 2$ stacking (upper figure). The rigid $\mathrm{C} 2$, in turn, inhibits the $\mathrm{ADP} / \mathrm{ATP}$ exchange in $\mathrm{C} 1$ while facilitating $\mathrm{ADP}$ accumulation. ADP-rich $\mathrm{C} 1$ is then transformed from $\mathrm{BI}$ to $\mathrm{BC}$ conformational state of $\mathrm{C} 1$, which induces the formation of the C1-KaiB-KaiA complex, i.e. the sequestration of KaiA from C2 (right figure). As KaiA no longer acts on C2 due to the sequestration, C2 dephosphorylation proceeds along with the conformational transition to the flexible conformational state of C2 (lower figure), which allows the ADP/ATP exchange in C1. Finally, after the release of ADP from C1, C1 returns to the BI conformational state and releases KaiB and KaiA (return to the left figure again).

Here, we make some remarks on KaiA and KaiB. All KaiA proteins are treated as dimers in this model. Although KaiB proteins are known to form monomers, dimers, and tetramers, all KaiB proteins are treated as monomers in this model for simplicity. Indeed, monomeric 
form is dominant near the so-called standard condition $(3.5 \mu \mathrm{M})[35]$. A recent experiment has shown that KaiB has two conformational states, and the transition between them plays a role in the slow KaiB-C1 binding[36]. In the present model, however, we consider only one conformational state of KaiB to focus more on the conformational transitions of KaiC and assume that the slowness arises from $\mathrm{C} 1$ rather than KaiB, as claimed by a recent experimental study[34].

\section{Parameter Optimization}

Next, as a validation of the present model, we show that the present model can quantitatively reproduce various experimental data. The parameter values are determined by an automatic optimization method that simultaneously fits multiple types of model outputs to corresponding experimental data. Here, we optimize 19 independent rate and equilibrium constants with an Arrhenius temperature dependence (38 parameters, i.e. their pre-exponential factor, the activation energy of rate constants, and the activation energy difference of equilibrium constants between corresponding forward and backward rate constants). The detailed procedure of the optimization, the experimental data used in the optimization, and the optimized parameter values are summarized in Method.

Figure 3 shows the results of the fitting. Figures 3A-D exhibit the phosphorylation oscillation of $\mathrm{KaiC}$ at $26-38{ }^{\circ} \mathrm{C}$. In these cases, the results of the model are in good agreement with the corresponding experimental data[37], which indicates that the temperature compensation of the period is realized in the present model. Figures $3 \mathrm{E}$ and $\mathrm{F}$ show the phosphorylation of KaiC in the absence of both KaiA and KaiB (Fig. 3E) and in the presence of KaiA (Fig. 3F), in which the experimental data of the $\mathrm{U}$ and $\mathrm{T}$ states in the former case[38] at time $t=20 \mathrm{~h}$ and the data of the four states in the latter case[8] at time $t=20$ $\mathrm{h}$ are used in the optimization. In these cases, the results of the model slightly deviate from the experimental data used in the optimization. However, relaxation behaviors, e.g. overshoots of the S state in the absence of KaiAB (Fig. 3E) and of the T state in the presence of KaiA (Fig. 3E), are well reproduced although the experimental data are adopted only from a time point $(t=20 \mathrm{~h})$. Figure $3 \mathrm{G}$ shows the ratio of the bound ATP in KaiC to the bound nucleotide, in which the corresponding experimental data[16] at time $t=8 \mathrm{~h}$ are considered in the optimization. Although the result of the model in the presence of KaiA 
deviates from the experimental data to some extent, the model reproduces the experimental result that the ratio in the presence of KaiA is larger than in the absence of KaiAB due to the promotion of the ADP/ATP exchange in C2 by KaiA[16]. Figure 3H shows the ATPase activities, i.e. the rates of ADP production, in the absence of KaiAB at $25-40{ }^{\circ} \mathrm{C}$. The result of the model exhibits the initial temperature dependence (thermal sensitivity $\mathrm{Q}_{10}=1.3$ at $t=0.1 \mathrm{~h})$ and the temperature compensation at the steady state $\left(Q_{10}=1.1\right.$ at $\left.t=\infty\right)$, which are consistent with the experimental results[22] used in the optimization $\left(\mathrm{Q}_{10}=1.4\right.$ and 1.1, respectively). Moreover, the present model can reproduce the overshoot of the ATPase activity[22] without additional experimental data. Figure 3I shows the total KaiA concentration dependence of the period in the present model, in which the experimental data[3] at the total concentration $\mathrm{A}_{\text {tot }}=3.6 \mu \mathrm{M}$ is used in the optimization. The period of the model is almost constant when $\mathrm{A}_{\text {tot }}<3.5 \mu \mathrm{M}$ as in the experiments. The total KaiB concentration dependence of the phosphorylation oscillation[3] is also well reproduced by the present model (Fig. 3J); the phosphorylation oscillation is almost independent of the total concentration $\mathrm{B}_{\text {tot }}$ when $\mathrm{B}_{\text {tot }}>1.8 \mu \mathrm{M}$, while the rhythmic behavior drastically disappears with decreasing in $\mathrm{B}_{\text {tot }}$ below $1.8 \mu \mathrm{M}$. Other results of the fitting are shown in Supplementary Information.

\section{Intra-KaiC unidirectional allostery}

Next, we investigate the origins of the clock functions of the KaiABC oscillator with the present model. In particular, we here focus on the unidirectional allostery from $\mathrm{C} 2$ to $\mathrm{C} 1$ in the present model, which is hypothetically introduced to realize the C1-independent C2 dephosphorylation[24, 25], and discuss the functional roles of the allostery on the generation of oscillation and on the period robustness against environmental perturbations.

Although the unidirectional allostery apparently contradicts with an intuition that C1 and C2 may bidirectionally interplay, the present model indeed shows that the unidirectional allostery is sufficient to generate a circadian rhythm. This sufficiency is assured by KaiA surrounding KaiC because $\mathrm{C} 1$ can indirectly communicate with $\mathrm{C} 2$ through the concentration of unbound KaiA, which is controlled by the sequestration of KaiA at C1 (Fig. 4); in the present model, information on phosphorylation is unidirectionally transmitted from $\mathrm{C} 2$ to $\mathrm{C} 1$ by the allostery of each KaiC hexamer, whereas information on phosphorylation/dephosphorylation 
switching is indirectly transmitted from $\mathrm{C} 1$ to $\mathrm{C} 2$ via the concentration of the free KaiA surrounding KaiC.

As discussed previously[26, 29], the "collectivity" of the KaiABC oscillator may be given by the "inter"-KaiC communication mediated by KaiA: phosphorylated KaiC sequesters KaiA and waits for other unphosphorylated KaiC to be phosphorylated; after free KaiA is decreased below a threshold due to the sequestration, dephosphorylation of KaiC occurs at once. We here make a remark that the unidirectionality of the allostery in each KaiC hexamer may have a positive role on an effective generation of the collective oscillation. If $\mathrm{C} 1$ and $\mathrm{C} 2$ bidirectionally interplay, the bidirectional communication could switch the oscillation phase within a hexamer independently of other hexamers, and hence, the waiting process mentioned above could be disrupted.

\section{Period robustness against the total concentration of KaiA}

As shown above, the present model can reproduce the period robustness against the total concentration of KaiA (Fig. 3I) and temperature (Fig. 3A-D). To explain possible mechanisms of the robustness in terms of the allosteric communication between $\mathrm{C} 1$ and $\mathrm{C} 2$, we here focus on the attenuation of $\mathrm{C} 1-\mathrm{KaiB}$ binding by KaiA acting on $\mathrm{C} 2$. This attenuation has been observed experimentally and was originally modeled through a global conformational transition of the whole $\mathrm{KaiC}[28]$, where $\mathrm{C} 1$ and $\mathrm{C} 2$ bidirectionally communicate. However, it is noted that this attenuation is also realized in the present model with only the unidirectional allostery. Specifically, KaiA binding to C2 stabilizes the flexible conformational state of $\mathrm{C} 2$ by shifting the equilibrium shown in Fig. 2B to right. This flexible state then triggers the $\mathrm{ADP} / \mathrm{ATP}$ exchange in $\mathrm{C} 1$, which prevents the KaiB binding to $\mathrm{C} 1$ as explained in the model setup.

To investigate the origin of the period robustness against the total concentration of KaiA, $\mathrm{A}_{\text {tot }}$, we first divide the period of phosphorylation oscillation into the durations of phosphorylation $\tau_{\mathrm{p}}$ and dephosphorylation $\tau_{\mathrm{dp}}$. Here $\tau_{\mathrm{p}}$ and dephosphorylation $\tau_{\mathrm{dp}}$ are defined by the period when more than 0.5 percent of KaiA binds to $\mathrm{C} 2$ or not, respectively. In the present model, $\tau_{\text {dp }}$ decreases, whereas $\tau_{\mathrm{p}}$ increases with increasing $\mathrm{A}_{\text {tot }}$ (Fig.3I).

The decrease of $\tau_{\mathrm{dp}}$ with increasing $\mathrm{A}_{\text {tot }}$ can be explained as follows. As suggested in many previous studies, KaiA is sufficiently sequestered from $\mathrm{C} 2$ by KaiB bound to $\mathrm{C} 1$ in 
the dephosphorylation phase. The oscillation then switches to the phosphorylation phase when the amount of KaiB bound to $\mathrm{C} 1$ falls below the threshold for the sufficient KaiA sequestration. Assuming this mechanism, the increase in $\mathrm{A}_{\text {tot }}$ lifts the threshold because KaiB must sequester more KaiA, and hence, the dephosphorylation phase terminates earlier.

On the other hand, the increase in $\tau_{\mathrm{p}}$ with increasing $\mathrm{A}_{\text {tot }}$ can be attributed to the attenuation of $\mathrm{C} 1-\mathrm{KaiB}$ binding by KaiA acting on $\mathrm{C} 2$ mentioned above. With increasing $\mathrm{A}_{\text {tot }}$, the attenuated KaiB binding to $\mathrm{C} 1$ delays the subsequent KaiA sequestration, and hence $\tau_{\mathrm{p}}$ becomes long.

The period robustness against KaiA concentration can be explained by a proper balance between the opposing effects on $\tau_{\mathrm{p}}$ and $\tau_{\mathrm{dp}}$. Indeed, with a modified parameter to disrupt the balance, the present model loses the period robustness (Figs. 5A and B). Specifically, an increase in the rate of $\mathrm{ADP} / \mathrm{ATP}$ exchange of $\mathrm{C} 1, k_{1 \mathrm{ex}}$, enhances the attenuation of KaiB binding by KaiA because the exchange inhibits the KaiB binding as explained in the model setup, and hence, $\tau_{\mathrm{p}}$ becomes more sensitive to $\mathrm{A}_{\text {tot }}$ change with increasing $k_{1 \mathrm{ex}}$ (Fig. 5B), and vice versa (Fig. 5A). The present model thus indicates that the proper balance is essential for the period robustness against $A_{\text {tot }}$.

\section{Period robustness against temperature}

Lastly, we discuss how the period robustness against temperature, i.e. the temperature compensation of the period, arises from the combination of elementary reactions. As experiments have shown that not only the period but also both $\tau_{\mathrm{p}}$ and $\tau_{\mathrm{dp}}$ are temperaturecompensated (Figs. 3A-D), we separately discuss $\tau_{\mathrm{p}}$ and $\tau_{\mathrm{dp}}$ again.

The temperature compensation of $\tau_{\mathrm{p}}$ in the present model can be explained by the balance between the following two opposing effects. On the one hand, $\tau_{\mathrm{p}}$ is shortened with increasing temperature because the phosphorylation reactions are accelerated due to their high activation energies (see Table I. For example, 30.0 and $36.5 \mathrm{kcal} / \mathrm{mol}$ for the phosphorylation reactions of Ser431 and Thr432 in the rigid C2, respectively). On the other hand, $\tau_{\mathrm{p}}$ is prolonged with increasing temperature because the attenuation of KaiB binding to $\mathrm{C} 1$ by KaiA is enhanced. The optimized parameters indeed show that the affinity between $\mathrm{C} 2$ and KaiA is strengthened when temperature increases; the equilibrium constant of $\mathrm{C} 2$ KaiA binding, $K_{2 \mathrm{~A}}$, decreases because its activation energy difference, i.e. coefficient of the 
temperature-dependent term in the exponent, is largely negative $(-32.4 \mathrm{kcal} / \mathrm{mol})$.

In the present model, an imbalance between the two opposing effects on $\tau_{\mathrm{p}}$ indeed leads to a loss of the temperature compensation of $\tau_{\mathrm{p}}$ (Figs. $5 \mathrm{C}$ and $\mathrm{D}$ ). We here modify the temperature dependence of $K_{2 \mathrm{~A}}$ to disrupt the balance. When $K_{2 \mathrm{~A}}$ is assumed to be independent of temperature (Fig. $5 \mathrm{C}$ ), the increase in $\tau_{\mathrm{p}}$ is lost. In this case, $\tau_{\mathrm{p}}$ decreases as temperature rises $\left(Q_{10}=0.67\right)$. On the other hand, when we amplify the temperature dependence of $K_{2 \mathrm{~A}}$ with a higher activation energy difference $(-80 \mathrm{kcal} / \mathrm{mol})$ (Fig. 5D), $\tau_{\mathrm{p}}$ increases with temperature $\left(Q_{10}=1.53\right)$. Thus, in the present model, the temperature compensation of $\tau_{\mathrm{p}}$ is described by the balance between the acceleration of phosphorylation reactions and the attenuation of $\mathrm{C} 1-\mathrm{KaiB}$ binding by KaiA.

The temperature compensation of $\tau_{\mathrm{dp}}$, on the other hand, can be explained through the ratio of the rate constants of phosphorylation to dephosphorylation reactions. Because the amplitude of the phosphorylation oscillation can be approximated by $\bar{k}_{\mathrm{p}} \tau_{\mathrm{p}}$ as well as by $\bar{k}_{\mathrm{dp}} \tau_{\mathrm{dp}}$, where $\bar{k}_{\mathrm{p}}$ and $\bar{k}_{\mathrm{dp}}$ are the mean rate constants of the phosphorylation and dephosphorylation reactions, respectively, we obtain

$$
\tau_{\mathrm{dp}} \sim\left(\bar{k}_{\mathrm{p}} / \bar{k}_{\mathrm{dp}}\right) \tau_{\mathrm{p}}
$$

As $\tau_{\mathrm{p}}$ is temperature-compensated, the temperature compensation of $\tau_{\mathrm{dp}}$ is achieved when $\bar{k}_{\mathrm{p}} / \bar{k}_{\mathrm{dp}}$ is also temperature-compensated, i.e. the activation energies of $\bar{k}_{\mathrm{p}}$ and $\bar{k}_{\mathrm{dp}}$ are in the same order. This is the case in the present model; for instance, the activation energies of phosphorylation reaction of Thr432 and dephosphorylation reaction of Ser431 in the rigid conformational state are 36.5 and $37.1 \mathrm{kcal} / \mathrm{mol}$, respectively. Note that the validity of the estimation in Eq. (1) can be alternatively confirmed by the results shown in Figs. 5C and D. With Eq. (1) and the temperature competition of $\bar{k}_{\mathrm{p}} / \bar{k}_{\mathrm{dp}}, \tau_{\mathrm{p}} / \tau_{\mathrm{dp}}$ is expected to be temperature-compensated even when each of $\tau_{\mathrm{p}}$ and $\tau_{\mathrm{dp}}$ depends on temperature. Indeed, $\tau_{\mathrm{p}} / \tau_{\mathrm{dp}}$ in Figs. $5 \mathrm{C}$ and $\mathrm{D}$ are relatively temperature-compensated $\left(Q_{10}=1.1\right.$ and 1.2 , respectively).

\section{DISCUSSION}

In this study, we developed a mathematical model of the circadian rhythm of Kai proteins consisting of the elementary reactions: chemical reactions of ligands, domain-specific 
conformational transitions, and (de)formation of complex among KaiABC. Most of the conventional mathematical models of the KaiABC oscillator have been conceptually simplified, i.e. represented by simplified processes rather than elementary reactions, to find universality in biological clocks. In contrast, the present model elucidate the microscopic origin of biological functions in the system by decomposing the behavior of the system into the elementary reactions.

The allosteric communication between $\mathrm{C} 1$ and $\mathrm{C} 2$ is crucial to generate the circadian rhythm. To investigate this allostery, we introduced the domain-specific conformational transitions in the present model, in contrast to a single global conformational transition of the whole KaiC assumed in the previous studies[26-29]. Importantly, we hypothetically designed the C2-dependent ADP/ATP exchange in C1 as the only explicit allosteric communication between $\mathrm{C} 1$ and $\mathrm{C} 2$. This allostery is unidirectional from $\mathrm{C} 2$ to $\mathrm{C} 1$, and thus explains the C1-independent dephosphorylation of C2[24, 25] without further assumptions. The present model reveals that the unidirectional allostery is sufficient for the generation of the oscillation and is important for the collectivity of the oscillation.

We further showed that the unidirectional allostery is sufficient to achieve the attenuation of $\mathrm{C} 1-\mathrm{KaiB}$ binding by the KaiA acting on $\mathrm{C} 2[28]$. In the present model, this attenuation yields the period robustness against both total KaiA concentration and temperature by adjusting the duration of the phosphorylation phase $\tau_{\mathrm{p}}$. This result means that the period robustness against the two very different factors may arise from a common molecular origin via the unidirectional allostery.

There have been several studies on the period robustness against the total KaiA concentration $[28,39]$, which have suggested that the robustness requires the multimerization of KaiC[28] and the phosphorylation-dependent affinity between KaiA and C2[39]. In this respect, the present model showed that these factors are insufficient to achieve the period robustness; the present model, which considers both multimerization and phosphorylationdependent affinity, can still lose the period robustness by disrupting the $\tau_{\mathrm{p}}$ adjustment. Our result suggests that a properly balanced $\tau_{\mathrm{p}}$ adjustment is also needed for the period robustness.

In the present study, we did not discuss the mechanism of entrainment to environmental changes, which is another fundamental property of biological clocks. This is to be discussed elsewhere. 


\section{METHODS}

\section{Multimeric forms of KaiABC in the present model}

KaiC in the present model takes a hexameric form. Both $\mathrm{C} 1$ and $\mathrm{C} 2$ domains have six ATP binding sites and undergo their own conformational transitions. The total concentration of KaiC hexamer $\left[\mathrm{C}_{6}\right]_{\text {tot }}$ is fixed to the so-called standard condition $(3.5 / 6 \mu \mathrm{M})$. KaiA and $\mathrm{KaiB}$ in the present model take dimeric and monomeric forms, respectively. Both KaiA and KaiB are assumed to have only one conformation. The total concentrations of KaiA dimer $\left[\mathrm{A}_{2}\right]_{\text {tot }}$ and KaiB monomer $[\mathrm{B}]_{\text {tot }}$ are fixed to the standard condition $(1.2 / 2 \mu \mathrm{M}$ and $3.5 \mu \mathrm{M}$, respectively).

\section{States of KaiC considered in the present model}

At each ATP binding site in C1, the ATP- and ADP-bound states are considered. On the other hand, states of ATP binding site in C2 are distinguished by phosphorylation of Ser431 and Thr432 and bound nucleotide. They are denoted by

$$
\mathrm{X}_{\mathrm{N}}(\mathrm{X} \in\{\mathrm{U}, \mathrm{S}, \mathrm{T}, \mathrm{D}\}, \mathrm{N} \in\{\mathrm{T}, \mathrm{D}\})
$$

where X represents a phosphorylation state, i.e. U (unphosphorylated), S (only Ser431 is phosphorylated ), T (only Thr432 is phosphorylated), or D (doubly phosphorylated), and subscript $\mathrm{N}$ represents a bound nucleotide, i.e. T (ATP) or D (ADP).

In the present model, one $\mathrm{C} 1$-specific and two $\mathrm{C} 2$-specific conformational transitions are considered. C1 undergoes a conformational transition between the KaiB-binding-competent (BC) and -incompetent (BI) conformational states. On the other hand, C2 undergoes conformational transitions between the rigid and flexible conformational states, and between the buried and exposed conformational states. Hence, four combinational states, i.e. rigidburied, rigid-exposed, flexible-buried, and flexible-exposed states are considered in C2 (in the present study, the rigid-exposed state will be omitted later).

In the present model, we considered all possible combination of the states listed above. The present model consists of a set of rate equations of elementary reactions that connect the combinational states. 


\section{Processes at each ATP binding site in $\mathrm{C} 1$ in the present model}

At each ATP binding site in C1, we consider ATP hydrolysis and ADP/ATP exchange. We assume that the phosphate release after the ATP hydrolysis is rapid and refer to the combination of these two processes simply as ATP hydrolysis. We also assume that the incorporation of exogenous ATP after the release of bound ADP is rapid and refer to the combination of these two processes as ADP/ATP exchange.

The rate constant of the ATP hydrolysis, $k_{1 \mathrm{~h}}$, is assumed to be independent of any KaiABC states for simplicity. On the other hand, the ADP/ATP exchange is allowed only when $\mathrm{C} 2$ is in the flexible state and $\mathrm{C} 1$ is in the BI state. We denote the rate constant of the ADP/ATP exchange in this case by $k_{1 \mathrm{ex}}^{\mathrm{BI} F}$ and in other case by $k_{1 \mathrm{ex}}^{\text {other }}$, which is fixed to 0 in the present study.

\section{Conformational transition of $\mathrm{C} 1$ in the present model}

We assume that the rate constants of the conformational transition from $\mathrm{BI}$ to $\mathrm{BC}$ state, $k_{1 \mathrm{IC}}$, depends on the number of bound ADP in $\mathrm{C} 1 n_{1 \mathrm{ADP}}$. Following the MonodWyman-Changeux model for multimerization effects[40] and the previous models for KaiABC oscillator[28, 29], we represent the dependence by the exponential form as

$$
k_{1 \mathrm{IC}}=k_{1 \mathrm{IC}, 0} \exp \left(n_{1 \mathrm{ADP}} \delta \mu_{1 \mathrm{ADP}}\right)
$$

where $k_{1 \mathrm{IC}, 0}$ is the rate constant when $\mathrm{C} 1$ fully binds $\mathrm{ATP}$, and $\delta \mu_{1 \mathrm{ADP}}$ is the factor for free energy difference. Moreover, $\delta \mu_{1 \mathrm{ADP}}$ is restricted to be positive to describe the promotion of the conformational transition from BI to BC state by the accumulation of ADP in C1.

On the other hand, we assume that the rate constants of the conformational transition from $\mathrm{BC}$ to $\mathrm{BI}$ state, $k_{1 \mathrm{CI}}$ is independent of any KaiABC states for simplicity.

\section{(De)formation of C1-KaiB-KaiA complex in the present model}

In the present model, at most six KaiB monomers can bind to $\mathrm{C} 1$ of a KaiC hexamer only when $\mathrm{C} 1$ is in the $\mathrm{BC}$ conformational state, and a KaiA dimer can bind to one of the KaiB monomers bound to $\mathrm{C} 1$. We assume that these bindings are much faster than other processes and adopt the rapid equilibrium approximation for these bindings. The equilibrium 
constants of the KaiB and KaiA bindings are denoted by $K_{1 \mathrm{~B}}$ and $K_{1 \mathrm{~A}}$, respectively. For simplicity, $K_{1 \mathrm{~B}}$ and $K_{1 \mathrm{~A}}$ are assumed to be independent of the number of KaiB bound to $\mathrm{C} 1$ or the number of KaiA bound to KaiB. Furthermore, the conformational transition of $\mathrm{C} 1$ from $\mathrm{BC}$ to $\mathrm{BI}$ state is assumed to occur regardless of the KaiAB binding, followed by the immediate release of the KaiA dimers and KaiB monomers on $\mathrm{C} 1$ as expressed by

$$
\mathrm{KaiC}_{6}(\mathrm{BC}) \cdot \mathrm{KaiB}_{n} \cdot \mathrm{KaiA}_{2 m} \stackrel{k_{1 \mathrm{CI}}}{\longrightarrow} \mathrm{KaiC}_{6}(\mathrm{BI})+n \mathrm{KaiB}+m \mathrm{KaiA}_{2} .
$$

Under the above assumptions, the total concentration of KaiC hexamer in the BC conformational state binding $n$ KaiB monomers, $\left[\mathrm{C}_{6}(\mathrm{BC}) \cdot \mathrm{B}_{n}\right]_{\text {tot }}$, is given by

$$
\left[\mathrm{C}_{6}(\mathrm{BC}) \cdot \mathrm{B}_{n}\right]_{\text {tot }}=\left(\begin{array}{c}
6 \\
n
\end{array}\right)\left(\frac{K_{1 \mathrm{~B}}}{[\mathrm{~B}]_{\text {free }}+K_{1 \mathrm{~B}}}\right)^{6-n}\left(\frac{[\mathrm{B}]_{\text {free }}}{[\mathrm{B}]_{\text {free }}+K_{1 \mathrm{~B}}}\right)^{n}\left[\mathrm{C}_{6}(\mathrm{BC})\right]_{\text {tot }},
$$

where $\left[\mathrm{C}_{6}(\mathrm{BC})\right]_{\text {tot }}$ is the total concentration of KaiC hexamer in the $\mathrm{BC}$ conformational state, and $[\mathrm{B}]_{\text {free }}$ is the concentration of unbound KaiB monomer. The concentration of $\mathrm{C}_{6}(\mathrm{BC}) \cdot \mathrm{B}_{n}$ binding $m$ KaiA dimers, $\left[\mathrm{C}_{6}(\mathrm{BC}) \cdot \mathrm{B}_{n} \cdot \mathrm{A}_{2 m}\right]$, is given by

$$
\left[\mathrm{C}_{6}(\mathrm{BC}) \cdot \mathrm{B}_{n} \cdot \mathrm{A}_{2 m}\right]=\left(\begin{array}{c}
n \\
m
\end{array}\right)\left(\frac{K_{1 \mathrm{~A}}}{\left[\mathrm{~A}_{2}\right]_{\text {free }}+K_{1 \mathrm{~A}}}\right)^{n-m}\left(\frac{\left[\mathrm{A}_{2}\right]_{\text {free }}}{\left[\mathrm{A}_{2}\right]_{\text {free }}+K_{1 \mathrm{~A}}}\right)^{m}\left[\mathrm{C}_{6}(\mathrm{BC}) \cdot \mathrm{B}_{n}\right]_{\text {tot }}
$$

where $\left[\mathrm{A}_{2}\right]_{\text {free }}$ is the concentration of unbound KaiA dimer. The total concentration of KaiB monomer bound to $\mathrm{C} 1,[\mathrm{~B}]_{\mathrm{onC} 1}$, is given by

$$
[\mathrm{B}]_{\mathrm{onC} 1}=\sum_{n=0}^{6} n\left[\mathrm{C}_{6}(\mathrm{BC}) \cdot \mathrm{B}_{n}\right]_{\text {tot }}=\frac{6[\mathrm{~B}]_{\text {free }}}{[\mathrm{B}]_{\text {free }}+K_{1 \mathrm{~B}}}\left[\mathrm{C}_{6}(\mathrm{BC})\right]_{\text {tot }} .
$$

Moreover, the total concentration of KaiA dimer bound to the KaiB-C1 complex is given by

$$
\left[\mathrm{A}_{2}\right]_{\mathrm{onC} 1}=\sum_{n=0}^{6} \sum_{m=0}^{n} m\left[\mathrm{C}_{6}(\mathrm{BC}) \cdot \mathrm{B}_{n} \cdot \mathrm{A}_{2 m}\right]=\frac{\left[\mathrm{A}_{2}\right]_{\text {free }}}{\left[\mathrm{A}_{2}\right]_{\text {free }}+K_{1 \mathrm{~A}}}[\mathrm{~B}]_{\mathrm{onC} 1} .
$$

Following the previous models of the KaiABC oscillator[8, 25, 28, 29], we assume that the bindings of KaiB and KaiA are very strong. In the present study, we fix both $K_{1 \mathrm{~B}}$ and $K_{1 \mathrm{~A}}$ to $1.0 \times 10^{-5} \mu \mathrm{M}$. Under the standard condition with this $K_{1 \mathrm{~B}}$, almost all KaiC hexamers in the $\mathrm{BC}$ conformational state bind six KaiB monomers.

\section{Processes at each ATP binding site in C2 in the present model}

At each ATP binding site in C2, we consider ATP hydrolysis and ADP/ATP exchange as in $\mathrm{C} 1$ (Fig. 1). Moreover, we consider phosphorylation and dephosphorylation reactions 
of Ser431 and Thr432 (Fig. 1), in which a phosphate is transferred between the bound nucleotide and either of Ser431 and Thr432.

For simplicity, we assume that the rate constant of the ATP hydrolysis, $k_{2 \mathrm{~h}}$, is independent of any KaiABC states. On the other hand, we assume the ADP/ATP exchange is allowed only when $\mathrm{C} 2$ is in the exposed conformational state. We denote the rate constant of the $\mathrm{ADP} / \mathrm{ATP}$ exchange in this case by $k_{2 \mathrm{ex}}^{\mathrm{E}}$ and when $\mathrm{C} 2$ is in the buried state by $k_{2 \mathrm{ex}}^{\mathrm{B}}$, which is fixed to 0 in the present study.

We assume that the rate constants of Ser431 phosphorylation and dephosphorylation between $\mathrm{U}_{\mathrm{T}}$ and $\mathrm{S}_{\mathrm{D}}\left(k_{\mathrm{S}+}\right.$ and $k_{\mathrm{S}-}$, respectively) depend on the rigid/flexible conformational states of $\mathrm{C} 2$ as well as the rate constants of the phosphorylation and dephosphorylation of Thr432 between $\mathrm{U}_{\mathrm{T}}$ and $\mathrm{T}_{\mathrm{D}}\left(k_{\mathrm{T}+}\right.$ and $k_{\mathrm{T}-}$, respectively). This dependence is explicitly expressed by superscripts $\mathrm{R}$ (rigid) and $\mathrm{F}$ (flexible) as in $k_{\mathrm{S}+}^{\mathrm{R}}$. For simplicity, the rate constants of the phosphorylation and dephosphorylation between $\mathrm{T}_{\mathrm{T}}$ and $\mathrm{D}_{\mathrm{D}}$ are assumed to be the same as those between $\mathrm{U}_{\mathrm{T}}$ and $\mathrm{S}_{\mathrm{D}}$, and those between $\mathrm{S}_{\mathrm{T}}$ and $\mathrm{D}_{\mathrm{D}}$ are also assumed to be the same as those between $\mathrm{U}_{\mathrm{T}}$ and $\mathrm{T}_{\mathrm{D}}$.

The all processes in each C2 ATP binding site are summarized in Eq. (9).

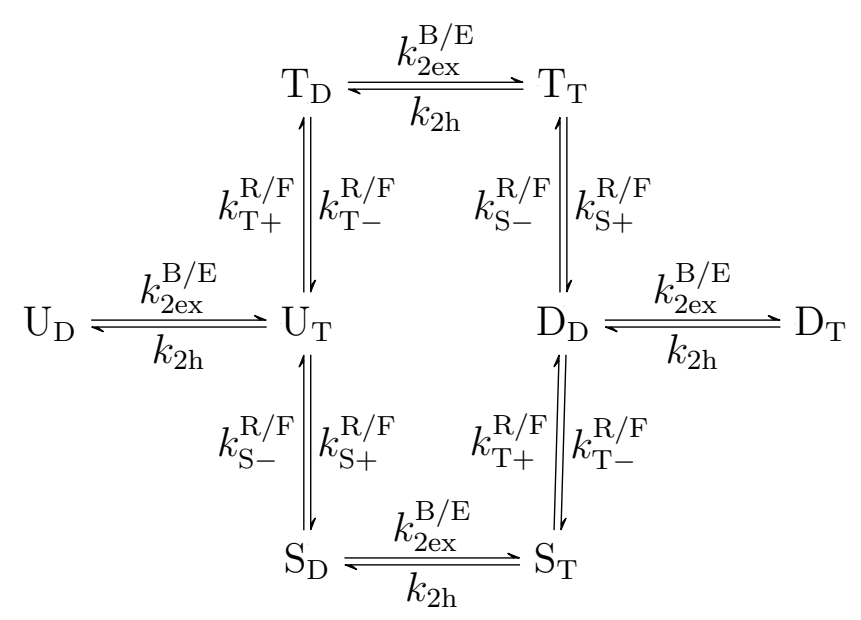

\section{Conformational transitions of C2 and KaiA binding to C2 in the present model}

In the present model, we assume that at most one KaiA dimer can bind to $\mathrm{C} 2$ of a KaiC hexamer only when $\mathrm{C} 2$ is in the exposed conformational state. We also assume that the KaiA binding to $\mathrm{C} 2$ and the two conformational transitions of $\mathrm{C} 2$ are much faster than other processes and adopt the rapid equilibrium approximation for these processes.

The equilibrium constant between the buried and exposed conformational states, $K_{2 \mathrm{~EB}}$, 
is assumed to depend on whether $\mathrm{C} 2$ is in the rigid or flexible conformational state $\left(K_{2 \mathrm{~EB}}^{\mathrm{R}}\right.$ and $K_{2 \mathrm{~EB}}^{\mathrm{F}}$, respectively). On the other hand, the equilibrium constant between the rigid and flexible conformational states, $K_{2 \mathrm{FR}}$, is assumed to depend on whether $\mathrm{C} 2$ is in the buried or exposed conformational state $\left(K_{2 \mathrm{FR}}^{\mathrm{B}}\right.$ and $K_{2 \mathrm{FR}}^{\mathrm{E}}$, respectively). Moreover, we assume that $K_{2 \mathrm{FR}}^{\mathrm{B}}$ and $K_{2 \mathrm{FR}}^{\mathrm{E}}$ depend on the phosphorylation-nucleotide states of $\mathrm{C} 2$. We represent the dependence by an exponential form as

$$
K_{2 \mathrm{FR}}^{\mathrm{B}}=K_{2 \mathrm{FR}, 0}^{\mathrm{B}} \exp \left(n_{2 \mathrm{pS}} \delta \mu_{2 \mathrm{pS}}+n_{2 \mathrm{pT}} \delta \mu_{2 \mathrm{pT}}+n_{2 \mathrm{ADP}} \delta \mu_{2 \mathrm{ADP}}\right)
$$

where $n_{2 \mathrm{pS}}, n_{2 \mathrm{pT}}$, and $n_{2 \mathrm{ADP}}$ denote the numbers of the phosphorylated Ser431 and Thr432, and the number of bound $\mathrm{ADP}$ in $\mathrm{C} 2$, respectively; $\delta \mu_{2 \mathrm{pS}}, \delta \mu_{2 \mathrm{pT}}$, and $\delta \mu_{2 \mathrm{ADP}}$ are the corresponding factor for free energy difference; and $K_{2 \mathrm{FR}, 0}^{\mathrm{B}}$ is the equilibrium constant when $\mathrm{C} 2$ is fully dephosphorylated and fully binds ATP. Note that the rigidification of C2 by the phosphorylation of Ser431 is described by positive $\delta \mu_{2 \mathrm{pS}}$. In the present model, $K_{2 \mathrm{FR}}^{\mathrm{E}}$ is determined by the detailed balance as explained below (Eq. (17)). For simplicity, we assume that the equilibrium constant of the KaiA binding to $\mathrm{C} 2, K_{2 \mathrm{~A}}$, as well as $K_{2 \mathrm{~EB}}^{\mathrm{R}}$ and $K_{2 \mathrm{~EB}}^{\mathrm{F}}$ is independent of the phosphorylation-nucleotide states of C2. Note that even under this assumption, the affinity between KaiA and C2 still depends on the phosphorylation-nucleotide states because the population of the exposed state, which is competent of KaiA binding, is controlled by the phosphorylation-nucleotide dependence of the rigid/flexible conformational transition.

The conformational transitions of $\mathrm{C} 2$ and KaiA binding to $\mathrm{C} 2$ are summarized in Eq. (11).

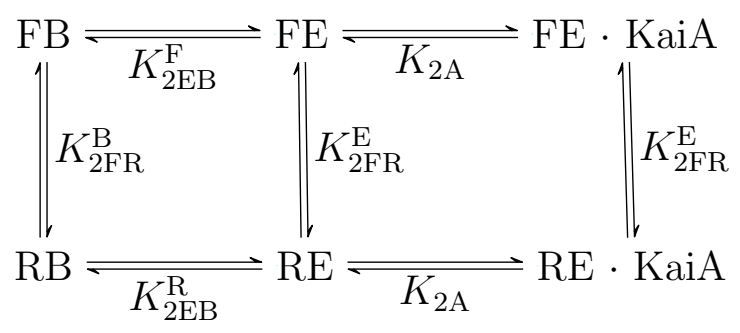

In the schematic representation described above, $X Y(X \in\{F, R\}, Y \in\{E, B\})$ represent the conformational state of $\mathrm{C} 2$, i.e. F, R, E, and B denote the flexible, rigid, exposed, and buried conformational states, respectively.

In the present study, we further assume that the exposed conformational state is absent 
when $\mathrm{C} 2$ is in the rigid state, that is

$$
K_{2 \mathrm{~EB}}^{R}=\infty
$$

In this case, the scheme in Eq. (11) is reduced to

$$
\mathrm{RB} \rightleftharpoons K_{2 \mathrm{FR}}^{\mathrm{B}} \mathrm{FB} \rightleftharpoons K_{2 \mathrm{~EB}}^{\mathrm{F}} \mathrm{FE} \rightleftharpoons K_{2 \mathrm{~A}} \mathrm{FE} \cdot \mathrm{KaiA}
$$

which is equivalent to the scheme shown in Fig. 2B. The total concentration of KaiA dimer bound to $\mathrm{C} 2$ is given by

$$
\left[\mathrm{A}_{2}\right]_{\mathrm{onC} 2}=\sum_{\mathrm{C} 2} \frac{\left[\mathrm{A}_{2}\right]_{\text {free }}}{\left[\mathrm{A}_{2}\right]_{\text {free }}+K_{2 \mathrm{~A}}\left(1+K_{2 \mathrm{~EB}}^{\mathrm{F}}\left(1+K_{2 \mathrm{FR}}^{\mathrm{B}}(\mathrm{C} 2)\right)\right)}\left[\mathrm{C}_{6}(\mathrm{C} 2)\right]_{\text {tot }},
$$

where $K_{2 \mathrm{FR}}^{\mathrm{B}}(\mathrm{C} 2)$ and $\left[\mathrm{C}_{6}(\mathrm{C} 2)\right]_{\text {tot }}$ are $K_{2 \mathrm{FR}}^{\mathrm{B}}$ and the total concentration of KaiC hexamer in a C2's phosphorylation-nucleotide state.

\section{Conservations of total KaiB monomer and KaiA dimer in the present model}

The conservations of the total concentrations of KaiB monomer $[\mathrm{B}]_{\text {tot }}$ and KaiA dimer $\left[\mathrm{A}_{2}\right]_{\text {tot }}$ are represented as

$$
\begin{aligned}
& {[\mathrm{B}]_{\text {tot }}=[\mathrm{B}]_{\text {free }}+[\mathrm{B}]_{\mathrm{onC} 1},} \\
& {\left[\mathrm{~A}_{2}\right]_{\text {tot }}=\left[\mathrm{A}_{2}\right]_{\text {free }}+\left[\mathrm{A}_{2}\right]_{\mathrm{onC} 1}+\left[\mathrm{A}_{2}\right]_{\mathrm{onC} 2} .}
\end{aligned}
$$

Thus, $[\mathrm{B}]_{\text {free }}$ and $\left[\mathrm{A}_{2}\right]_{\text {free }}$ can be obtained by solving Eqs. (15) and (16) with Eqs. (7), (8), and (14).

\section{Constraints arising from the detailed balance in the present model}

To assure thermodynamic consistency, the present model imposes the detailed balance condition on the processes constituting a loop. As in Paijmans's model[29], we omit the ATP hydrolysis and the ADP/ATP exchange from the processes subjected to the detailed balance. Note that the allosteric communication between $\mathrm{C} 1$ and $\mathrm{C} 2$ in the present model is unrelated to the detailed balance because the present allostery is represented only by the rate constant of $\mathrm{ADP} / \mathrm{ATP}$ exchange in $\mathrm{C} 1$. 
For the loop consisting of the conformational transitions between the rigid and flexible conformational states and between the buried and exposed conformational states (Eq. (11)), the detailed balance is given by

$$
K_{2 \mathrm{FR}}^{\mathrm{E}}=\frac{K_{2 \mathrm{~EB}}^{\mathrm{F}}}{K_{2 \mathrm{~EB}}^{\mathrm{R}}} K_{2 \mathrm{FR}}^{\mathrm{R}}
$$

In the present model, there exists a loop consisting of the (de)phosphorylation of Ser431 and the conformational transitions between the rigid and flexible conformational states as shown in Eq. (18).

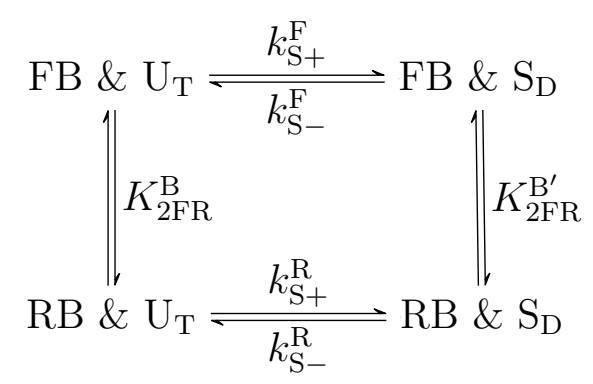

The detailed balance for this loop is

$$
\frac{k_{\mathrm{S}+}^{\mathrm{R}}}{k_{\mathrm{S}-}^{\mathrm{R}}}=\frac{K_{2 \mathrm{FR}}^{\mathrm{B}^{\prime}}}{K_{2 \mathrm{FR}}^{\mathrm{B}}} \frac{k_{\mathrm{S}+}^{\mathrm{F}}}{k_{\mathrm{S}-}^{\mathrm{F}}}=\exp \left(\delta \mu_{2 \mathrm{pS}}+\delta \mu_{2 \mathrm{ADP}}\right) \frac{k_{\mathrm{S}+}^{\mathrm{F}}}{k_{\mathrm{S}-}^{\mathrm{F}}} .
$$

Similarly, the detailed balance about Thr432 is expressed as

$$
\frac{k_{\mathrm{T}+}^{\mathrm{R}}}{k_{\mathrm{T}-}^{\mathrm{R}}}=\exp \left(\delta \mu_{2 \mathrm{pT}}+\delta \mu_{2 \mathrm{ADP}}\right) \frac{k_{\mathrm{T}+}^{\mathrm{F}}}{k_{\mathrm{T}-}^{\mathrm{F}}} .
$$

\section{Temperature dependence of parameters in the present model}

We assume an Arrhenius temperature dependence for all the constants in the present model. The rate and equilibrium constants are represented by two parameters, the preexponential factor determined at $30{ }^{\circ} \mathrm{C}$ and the activation energy for a rate constant or the activation energy difference for an equilibrium constant. A rate constant $k(T)$ and an equilibrium constant $K(T)$ are represented as

$$
\begin{array}{r}
k(T)=k_{30} \exp \left[-\frac{E_{\mathrm{a}}}{k_{\mathrm{B}}}\left(\frac{1}{T}-\frac{1}{T_{30}}\right)\right], \\
K(T)=K_{30} \exp \left[-\frac{\Delta E_{\mathrm{a}}}{k_{\mathrm{B}}}\left(\frac{1}{T}-\frac{1}{T_{30}}\right)\right],
\end{array}
$$

respectively, where $E_{\mathrm{a}}$ is the activation energy of the rate constant, $\Delta E_{\mathrm{a}}$ is the activation energy difference of the equilibrium constant, $k_{30}$ and $K_{30}$ are the values at $30{ }^{\circ} \mathrm{C}$ of the 
corresponding constants, $T_{30}$ is the temperature at $30{ }^{\circ} \mathrm{C}(=303.15 \mathrm{~K})$, and $k_{\mathrm{B}}$ is the Boltzmann constant. Factors for free energy difference in Eqs. (3) and (10) are represented by

$$
\delta \mu(T)=\delta \mu_{30}-\frac{\Delta E_{\mathrm{a}}}{k_{\mathrm{B}}}\left(\frac{1}{T}-\frac{1}{T_{30}}\right),
$$

where $\delta \mu_{30}$ is the value $\delta \mu$ at $30^{\circ} \mathrm{C}$.

\section{Parameter Optimization}

Optimization of the rate and equilibrium constants in the present model is performed to reproduce several experimental data including their temperature dependence. The parameter optimization is conducted through an automatic minimization of the difference between the experimental data and the outputs of the model. The loss function to be optimized considers multiple types of incorporates data simultaneously as

$$
L(\mathbf{k}, \mathbf{K}, \delta \mu)=\sum_{i, j} w_{i j}\left[x_{i}\left(t_{i j} ; \mathbf{k}, \mathbf{K}, \delta \mu\right)-x_{i, \exp }\left(t_{i j}\right)\right]^{2},
$$

where $x_{i, \exp }\left(t_{i j}\right)$ and $x_{i}\left(t_{i j} ; \mathbf{k}, \mathbf{K}, \delta \mu\right)$ denote the $i$ th experimental data at time $t_{i j}$ and the corresponding output of the model with parameters $\mathbf{k}, \mathbf{K}$, and $\delta \mu$, respectively, and $w_{i j}$ is the weight.

In the present study, the term $x_{i}\left(t_{i j} ; \mathbf{k}, \mathbf{K}, \delta \mu\right)$ was obtained through the integration of the rate equations by the fourth-order Runge-Kutta method, and the loss function was automatically minimized by the Nelder-Mead method.

The following experimental data were used in the parameter optimization:

- Phosphorylation in the presence of KaiAB at $26-40{ }^{\circ} \mathrm{C}$ (every $4{ }^{\circ} \mathrm{C}$ ) from time $t=0 \mathrm{~h}$ to $t=120 \mathrm{~h}$ (every $3 \mathrm{~h})[37]$;

- Phosphorylation in the presence of KaiA at $30{ }^{\circ} \mathrm{C}$ at time $t=20 \mathrm{~h}[8]$;

- Phosphorylation (only $\mathrm{U}$ and $\mathrm{T}$ states) in the absence of KaiAB at $30{ }^{\circ} \mathrm{C}$ at time $t=20 \mathrm{~h}[38]$

- Ratio of ATP in bound nucleotide (only maximum and minimum values) in the presence of KaiAB at $30{ }^{\circ} \mathrm{C}$ [16]; 
- Ratio of ATP in bound nucleotide in the presence of KaiA at $30{ }^{\circ} \mathrm{C}$ at time $t=8 \mathrm{~h}$ $[16]$

- Ratio of ATP in bound nucleotide in the absence of KaiAB at $30{ }^{\circ} \mathrm{C}$ at time $t=8 \mathrm{~h}$ $[16]$

- The maximum and minimum values of ATPase activity (i.e., rate of ADP production) in the presence of $\mathrm{KaiAB}$ at $30{ }^{\circ} \mathrm{C}[41]$;

- ATPase activity in the presence of KaiA at $30{ }^{\circ} \mathrm{C}$ at time $t=\infty[41]$;

- ATPase activity in the absence of KaiAB at $30{ }^{\circ} \mathrm{C}$ at time $t=\infty[41]$;

- Q10 values of ATPase activity in the absence of KaiAB at time $t=0.1$ and $t=\infty$ $[22]$

- Minimum value of $[\mathrm{B}]_{\text {free }}$ (in the presence of KaiAB at $30{ }^{\circ} \mathrm{C}$ ) restrained to $0.38 \mu \mathrm{M}$ to reproduce the arrhythmic/rhythmic phase transition around $[\mathrm{B}]_{\text {tot }} \sim 1.75 \mu \mathrm{M}[3]$; and

- Period when $[\mathrm{A}]_{\text {tot }} \sim 3.6 \mu \mathrm{M}[3]$.

In the calculation of the phosphorylation oscillation at various temperatures, the initial state of KaiC was set to the steady state $(t=\infty)$ of KaiC in the absence of KaiAB at $0{ }^{\circ} \mathrm{C}$.

The optimized parameters are shown in Table I.

\section{DATA AVAILABILITY}

All data generated in this study are available from the corresponding authors upon request.

[1] Ishiura, M. et al. Expression of a gene cluster kaiabc as a circadian feedback process in cyanobacteria. Science 281, 1519-1523 (1998). URL http://dx.doi.org/10.1126/science. 281.5382 .1519 . 
[2] Nakajima, M. et al. Reconstitution of circadian oscillation of cyanobacterial kaic phosphorylation in vitro. Science 308, 414-415 (2005). URL http://dx.doi.org/10.1126/science. 1108451.

[3] Nakajima, M., Ito, H. \& Kondo, T. In vitro regulation of circadian phosphorylation rhythm of cyanobacterial clock protein kaic by kaia and kaib. FEBS Letters 584, 898-902 (2010). URL http://dx.doi.org/10.1016/j.febslet.2010.01.016.

[4] Yoshida, T., Murayama, Y., Ito, H., Kageyama, H. \& Kondo, T. Nonparametric entrainment of the in vitro circadian phosphorylation rhythm of cyanobacterial KaiC by temperature cycle. Proceedings of the National Academy of Sciences 106, 1648-1653 (2009). URL http://dx. doi.org/10.1073/pnas.0806741106.

[5] Rust, M. J., Golden, S. S. \& O'Shea, E. K. Light-Driven Changes in Energy Metabolism Directly Entrain the Cyanobacterial Circadian Oscillator. Science 331, 220-223 (2011). URL http://dx.doi.org/10.1126/science.1197243.

[6] Mori, T. et al. Circadian clock protein kaic forms atp-dependent hexameric rings and binds dna. Proceedings of the National Academy of Sciences 99, 17203-17208 (2002). URL http: //dx.doi.org/10.1073/pnas.262578499.

[7] Hayashi, F. et al. Atp-induced hexameric ring structure of the cyanobacterial circadian clock protein kaic. Genes to Cells 8, 287-296 (2003). URL http://dx.doi.org/10.1046/j. 1365-2443.2003.00633.x.

[8] Rust, M. J., Markson, J. S., Lane, W. S., Fisher, D. S. \& O’Shea, E. K. Ordered phosphorylation governs oscillation of a three-protein circadian clock. Science 318, 809-812 (2007). URL http://dx.doi.org/10.1126/science.1148596.

[9] Nishiwaki, T. et al. A sequential program of dual phosphorylation of kaic as a basis for circadian rhythm in cyanobacteria. The EMBO Journal 26, 4029-4037 (2007). URL http: //dx.doi.org/10.1038/sj.emboj.7601832.

[10] Iwasaki, H., Nishiwaki, T., Kitayama, Y., Nakajima, M. \& Kondo, T. Kaia-stimulated kaic phosphorylation in circadian timing loops in cyanobacteria. Proceedings of the National Academy of Sciences 99, 15788-15793 (2002). URL http://dx.doi.org/10.1073/pnas. 222467299.

[11] Nishiwaki, T. et al. Role of kaic phosphorylation in the circadian clock system of synechococcus elongatus pcc 7942. Proceedings of the National Academy of Sciences of the United States of 
America 101, 13927-13932 (2004). URL http://dx.doi.org/10.1073/pnas.0403906101.

[12] Vakonakis, I. \& LiWang, A. C. Structure of the c-terminal domain of the clock protein kaia in complex with a kaic-derived peptide: Implications for kaic regulation. Proceedings of the National Academy of Sciences of the United States of America 101, 10925-10930 (2004). URL http://dx.doi.org/10.1073/pnas.0403037101.

[13] Pattanayek, R. et al. Visualizing a circadian clock protein crystal structure of kaic and functional insights. Molecular Cell 15, 375-388 (2004). URL http://dx.doi.org/10.1016/ j.molcel.2004.07.013.

[14] Kim, Y.-I., Dong, G., Carruthers, C. W., Golden, S. S. \& LiWang, A. The day/night switch in kaic, a central oscillator component of the circadian clock of cyanobacteria. Proceedings of the National Academy of Sciences 105, 12825-12830 (2008). URL http://dx.doi.org/10. 1073/pnas. 0800526105.

[15] Tseng, R. et al. Cooperative kaia-kaib-kaic interactions affect kaib/sasa competition in the circadian clock of cyanobacteria. Journal of Molecular Biology 426, 389-402 (2014). URL http://dx.doi.org/10.1016/j.jmb.2013.09.040.

[16] Nishiwaki-Ohkawa, T., Kitayama, Y., Ochiai, E. \& Kondo, T. Exchange of adp with atp in the cii atpase domain promotes autophosphorylation of cyanobacterial clock protein kaic. Proceedings of the National Academy of Sciences 111, 4455-4460 (2014). URL http://dx. doi.org/10.1073/pnas.1319353111.

[17] Kitayama, Y., Iwasaki, H., Nishiwaki, T. \& Kondo, T. Kaib functions as an attenuator of kaic phosphorylation in the cyanobacterial circadian clock system. The EMBO Journal 22, 2127-2134 (2003). URL http://dx.doi.org/10.1093/emboj/cdg212.

[18] Xu, Y., Mori, T. \& Johnson, C. H. Cyanobacterial circadian clockwork: roles of kaia, kaib and the kaibc promoter in regulating kaic. The EMBO Journal 22, 2117-2126 (2003). URL http://dx.doi.org/10.1093/emboj/cdg168.

[19] Chang, Y.-G., Tseng, R., Kuo, N.-W. \& LiWang, A. Rhythmic ring-ring stacking drives the circadian oscillator clockwise. Proceedings of the National Academy of Sciences 109, 1684716851 (2012). URL http://dx.doi.org/10.1073/pnas.1211508109.

[20] Snijder, J. et al. Structures of the cyanobacterial circadian oscillator frozen in a fully assembled state. Science 355, 1181-1184 (2017). URL http://dx .doi .org/10.1126/science . aag3218. 
[21] Tseng, R. et al. Structural basis of the day-night transition in a bacterial circadian clock. Science 355, 1174-1180 (2017). URL http://dx.doi.org/10.1126/science.aag2516.

[22] Abe, J. et al. Atomic-scale origins of slowness in the cyanobacterial circadian clock. Science 349, 312-316 (2015). URL http://dx.doi.org/10.1126/science.1261040.

[23] Chang, Y.-G., Kuo, N.-W., Tseng, R. \& LiWang, A. Flexibility of the c-terminal, or cii, ring of kaic governs the rhythm of the circadian clock of cyanobacteria. Proceedings of the National Academy of Sciences 108, 14431-14436 (2011). URL http://dx.doi.org/10.1073/pnas. 1104221108.

[24] Kitayama, Y., Nishiwaki-Ohkawa, T., Sugisawa, Y. \& Kondo, T. Kaic intersubunit communication facilitates robustness of circadian rhythms in cyanobacteria. Nature Communications 4 (2013). URL http://dx.doi.org/10.1038/ncomms3897.

[25] Phong, C., Markson, J. S., Wilhoite, C. M. \& Rust, M. J. Robust and tunable circadian rhythms from differentially sensitive catalytic domains. Proceedings of the National Academy of Sciences 110, 1124-1129 (2013). URL http://dx.doi.org/10.1073/pnas.1212113110.

[26] Zon, J. S. v., Lubensky, D. K., Altena, P. R. \& Wolde, P. t. An allosteric model of circadian kaic phosphorylation. Proceedings of the National Academy of Sciences 104, 7420-7425 (2007). URL http://dx.doi.org/10.1073/pnas.0608665104.

[27] Clodong, S. et al. Functioning and robustness of a bacterial circadian clock. Molecular Systems Biology 3 (2007). URL http://dx.doi.org/10.1038/msb4100128.

[28] Lin, J., Chew, J., Chockanathan, U. \& Rust, M. J. Mixtures of opposing phosphorylations within hexamers precisely time feedback in the cyanobacterial circadian clock. Proceedings of the National Academy of Sciences 111, E3937-E3945 (2014). URL http://dx.doi.org/10. 1073/pnas. 1408692111.

[29] Paijmans, J., Lubensky, D. K. \& Wolde, P. R. t. A thermodynamically consistent model of the post-translational kai circadian clock. PLOS Computational Biology 13, e1005415 (2017). URL http://dx.doi.org/10.1371/journal.pcbi.1005415.

[30] Egli, M. et al. Dephosphorylation of the core clock protein kaic in the cyanobacterial kaiabc circadian oscillator proceeds via an atp synthase mechanism. Biochemistry 51, 1547-1558 (2012). URL http://dx.doi.org/10.1021/bi201525n.

[31] Nishiwaki, T. \& Kondo, T. Circadian autodephosphorylation of cyanobacterial clock protein kaic occurs via formation of atp as intermediate. Journal of Biological Chemistry 287, 18030- 
18035 (2012). URL http://dx.doi.org/10.1074/jbc.m112.350660.

[32] Mutoh, R., Nishimura, A., Yasui, S., Onai, K. \& Ishiura, M. The atp-mediated regulation of kaib-kaic interaction in the cyanobacterial circadian clock. PLoS ONE 8, e80200 (2013). URL http://dx.doi.org/10.1371/journal.pone.0080200.

[33] Oyama, K., Azai, C., Nakamura, K., Tanaka, S. \& Terauchi, K. Conversion between two conformational states of kaic is induced by atp hydrolysis as a trigger for cyanobacterial circadian oscillation. Scientific Reports 6, 32443 (2016). URL http://dx.doi.org/10.1038/ srep32443.

[34] Mukaiyama, A. et al. Conformational rearrangements of the c1 ring in kaic measure the timing of assembly with kaib. Scientific Reports 8, 8803 (2018). URL http://dx.doi.org/10.1038/ s41598-018-27131-8.

[35] Snijder, J. et al. Insight into cyanobacterial circadian timing from structural details of the kaib-kaic interaction. Proceedings of the National Academy of Sciences 111, 1379-1384 (2014). URL http://dx.doi.org/10.1073/pnas.1314326111.

[36] Chang, Y.-G. et al. A protein fold switch joins the circadian oscillator to clock output in cyanobacteria. Science 349, 324-328 (2015). URL http://dx.doi.org/10.1126/science. 1260031.

[37] Furuike, Y., Abe, J., Mukaiyama, A. \& Akiyama, S. Accelerating in vitro studies on circadian clock systems using an automated sampling device. Biophysics and Physicobiology 13, 235-241 (2016). URL http://dx.doi.org/10.2142/biophysico.13.0_235.

[38] Murayama, Y. et al. Tracking and visualizing the circadian ticking of the cyanobacterial clock protein kaic in solution. The EMBO Journal 30, 68-78 (2011). URL http://dx.doi.org/ 10.1038/emboj.2010.298.

[39] Mori, T. et al. Revealing circadian mechanisms of integration and resilience by visualizing clock proteins working in real time. Nature Communications 9, 3245 (2018). URL http: //dx.doi.org/10.1038/s41467-018-05438-4.

[40] Monod, J., Wyman, J. \& Changeux, J.-P. On the nature of allosteric transitions: A plausible model. Journal of Molecular Biology 12, 88-118 (1965). URL http://dx.doi.org/10.1016/ s0022-2836(65) 80285-6.

[41] Terauchi, K. et al. Atpase activity of kaic determines the basic timing for circadian clock of cyanobacteria. Proceedings of the National Academy of Sciences 104, 16377-16381 (2007). 
URL http://dx.doi.org/10.1073/pnas.0706292104.

\section{ACKNOWLEDGEMENTS}

The authors are grateful to Y. Fruike, J. Abe, A. Mukaiyama, and S. Akiyama for the provision of raw experimental data in Ref. [37]. This work has been supported by JSPS KAKENHI, Grant Number JP18K14185 (SK) and JP16H02254 (SS), and the Indo-Japan bilateral collaboration program. The calculations were partially carried out on computers at the Research Center for Computational Science, Okazaki, Japan.

\section{AUTHOR CONTRIBUTIONS}

S.K. and S.S. designed research. S.K. performed research. S.K. and S.S. analyzed data and wrote the paper.

\section{COMPETING INTERESTS}

The authors declare no competing interests.

\section{ADDITIONAL INFORMATION}

Supplementary information is available for this paper. 
(A)
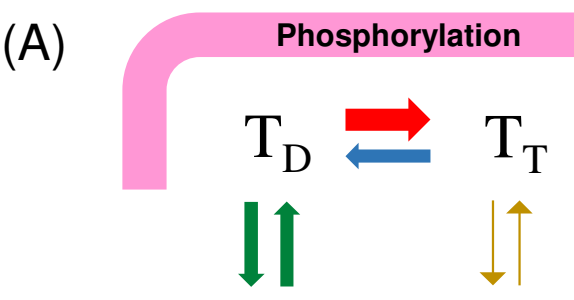

$\mathrm{U}_{\mathrm{D}}$

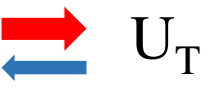

14

$\mathrm{S}_{\mathrm{D}}$

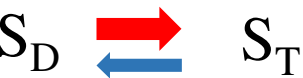

(B)

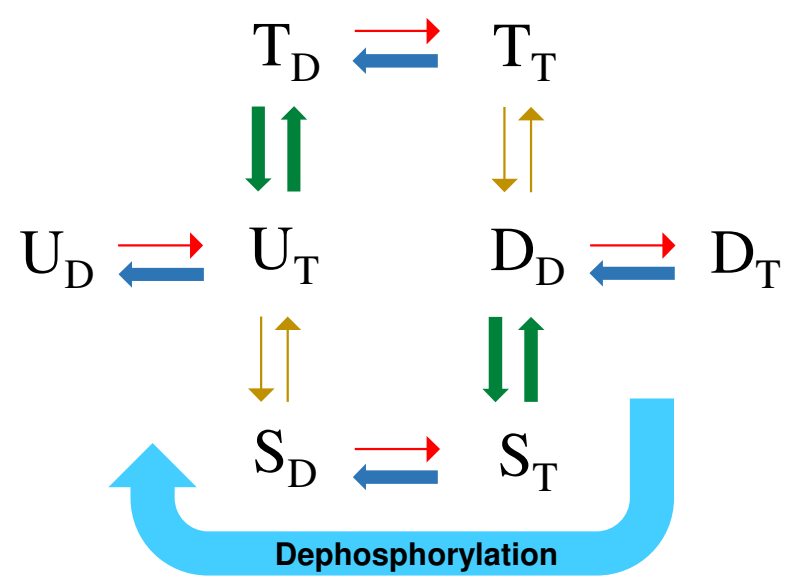

Thr (de)phos.

FIG. 1. Reaction scheme at a C2 ATP binding site. Red and blue arrows represent the ADP/ATP exchange and ATP hydrolysis (with phosphate release), and ocher and green arrows represent (de)phosphorylation of Ser431 and Thr432, respectively. Arrow thickness qualitatively represents the magnitude of a rate constant. The ADP/ATP exchange is the largest in (A) and the smallest in $(\mathrm{B})$. 


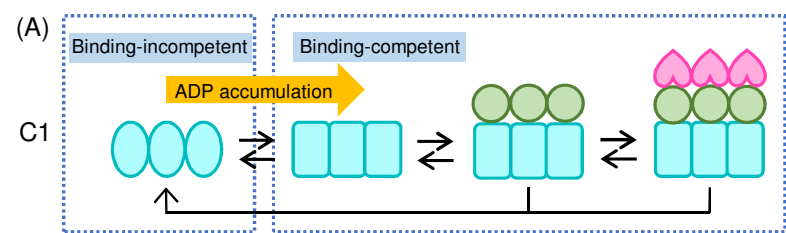

(B)

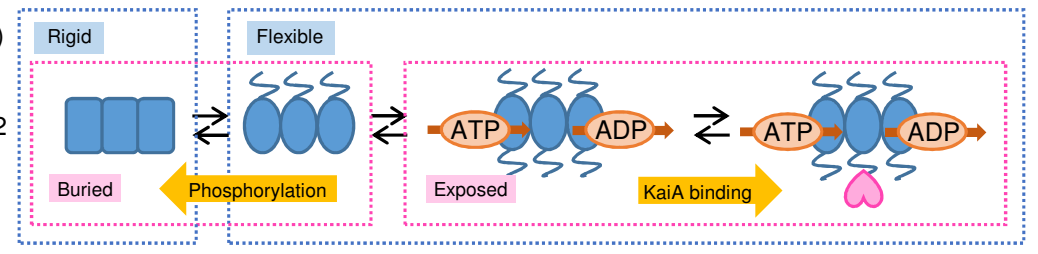

(C)

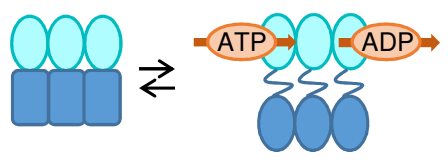

(D)

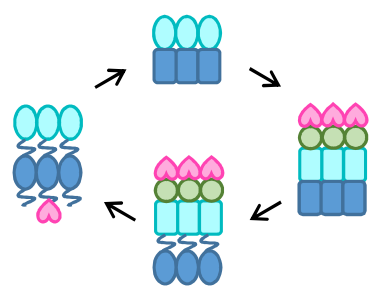

FIG. 2. Conformational transitions of KaiC in the present model. (A) Conformational transition of C1. Schematic figures of $\mathrm{C} 1$ with elliptical and rectangular shapes represent KaiB-bindingincompetent (BI) and -competent (BC) conformational states, respectively. KaiB can bind to C1 in the $\mathrm{BC}$ state, and KaiA can bind to KaiB bound to C1. (B) Conformational transitions of C2. Schematic figures of $\mathrm{C} 2$ with rectangular and elliptical shapes represent rigid and flexible conformational states of C2, respectively. Strings drawn from the top of C2 (linkers between C1 and $\mathrm{C} 2$ ) correspond to $\mathrm{C} 1-\mathrm{C} 2$ stacking, which is assumed to occur along with $\mathrm{C} 2$ rigidification. Strings drawn from the bottom of C2 (C-terminal tails) represent buried and exposed conformational states of $\mathrm{C} 2$. The exposed state, which is absent in the rigid state, can bind KaiA and allows ADP/ATP exchange in C2. (C) Allosteric communication in KaiC. C2 in the rigid state prohibits the ADP/ATP exchange in $\mathrm{C} 1$ due to the rigidification of $\mathrm{C} 2$. (D) Cycle of conformational transitions of KaiC during phosphorylation oscillation. 

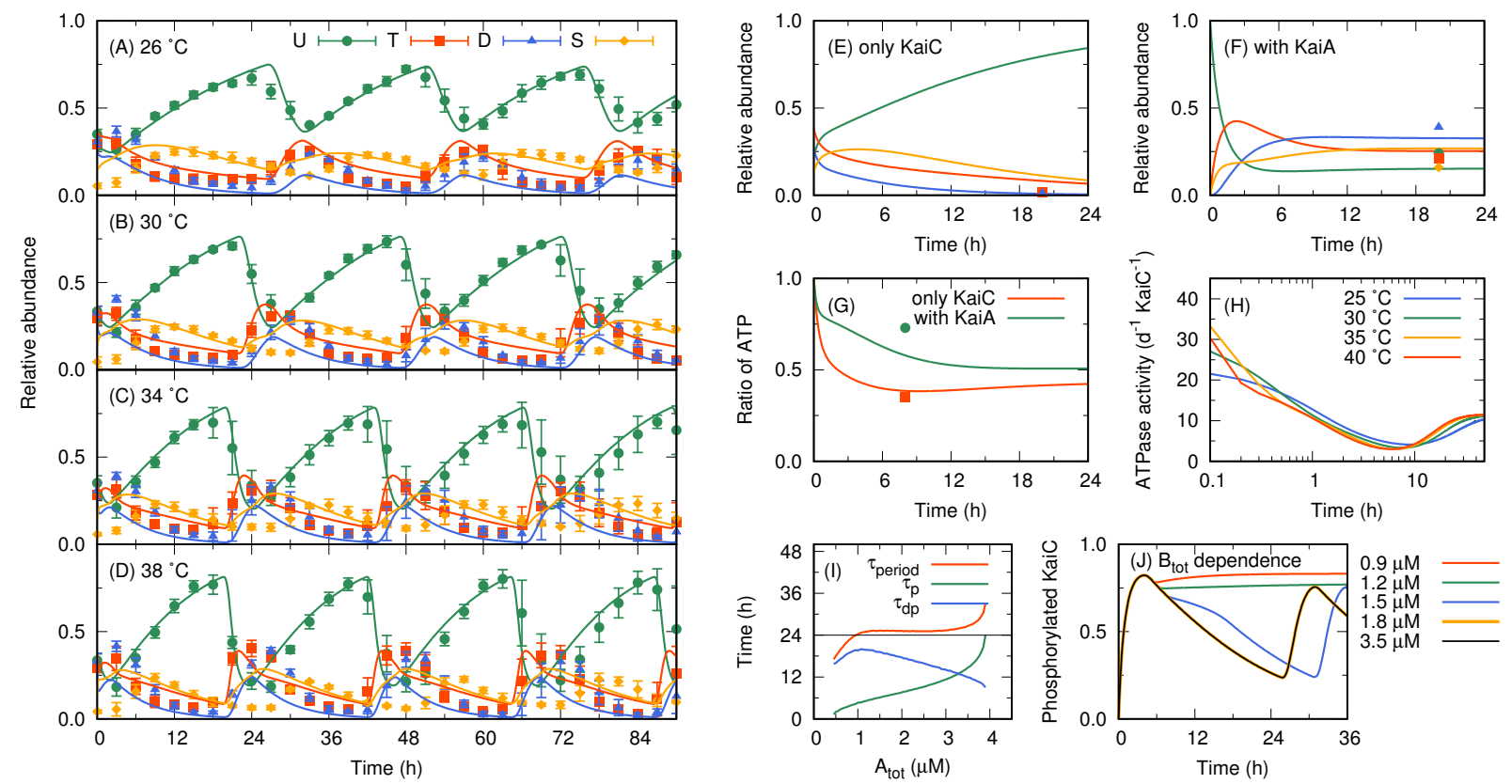

FIG. 3. Results of the present model with optimized parameters. Solid curves and dots represent the results of the model and the experimental data used in the parameter optimization. (A-F) Relative abundance of phosphorylation states (U, T, D, and S) of KaiC. (A-D) show the relative abundance of the system in the presence of KaiAB at 26, 30, 34, and $38^{\circ} \mathrm{C}$. Dots and error bars indicate the experimental data and their standard deviations[37], respectively. The initial state of the model is the steady state in the absence of KaiAB at $0{ }^{\circ} \mathrm{C}$. (E) shows the relative abundance of the system in the absence of $\mathrm{KaiAB}$ at $30{ }^{\circ} \mathrm{C}$ with the same initial state as that used in (AD). The experimental data are taken from Ref. [38]. (F) shows the relative abundance of the system in the presence of KaiA at $30{ }^{\circ} \mathrm{C}$. The initial state is fully dephosphorylated and binds ATP. The experimental data are taken from Ref. [8]. (G) Ratios of bound ATP in KaiC to bound nucleotide at $30{ }^{\circ} \mathrm{C}$ in the absence of KaiAB and in the presence of KaiA. The initial state is the same as that used in (F). The experimental data are taken from Ref. [16]. (H) Temperature dependence of ATPase activity in the absence of KaiAB. The initial phosphorylation state of the model is the steady state in the absence of KaiAB at $0{ }^{\circ} \mathrm{C}$, but all bond nucleotides are replaced to ATP. The experimental result of thermal sensitivities $Q_{10}$ at time $t=0.1 \mathrm{~h}$ and $\infty[22]$ are used in the optimization (not shown). (I) $\mathrm{A}_{\text {tot }}$ dependences of the period $\tau_{\text {period }}$, the duration of phosphorylation $\tau_{\mathrm{p}}$, and that of dephosphorylation $\tau_{\mathrm{dp}}$ at $30{ }^{\circ} \mathrm{C}$. $(\mathrm{J}) \mathrm{B}_{\text {tot }}$ dependence of the phosphorylation oscillation. The initial state is the same as that used in (F). The experimental data from Ref. [3] are used in the optimization to obtain (F) and (J) (Method). 


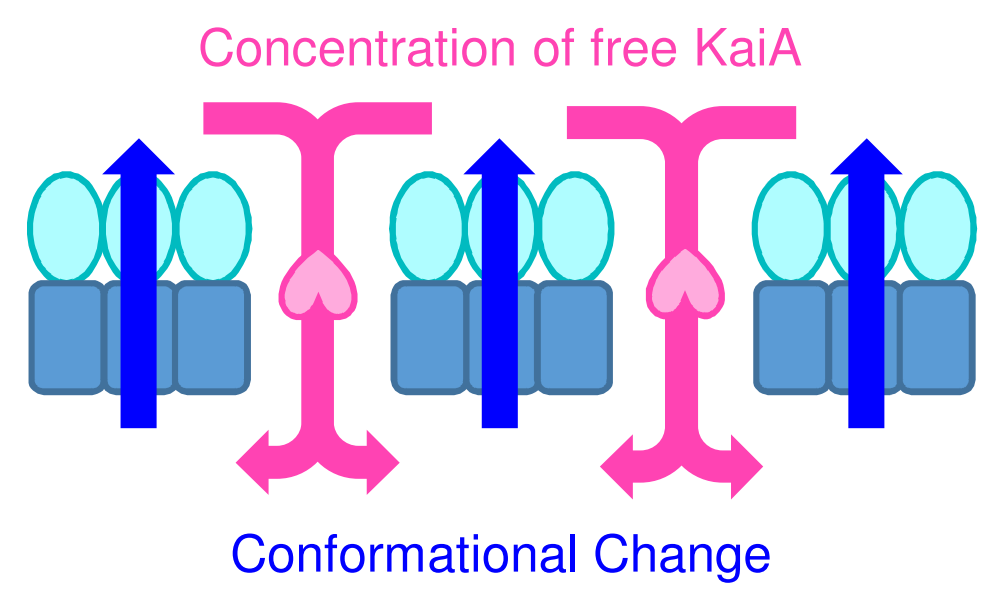

FIG. 4. Direction of information transmission in the present model. Information on the phosphorylation state is transmitted from $\mathrm{C} 2$ to $\mathrm{C} 1$ by conformational transitions of each KaiC hexamer (blue arrows), whereas information on phosphorylation/dephosphorylation switching is indirectly transmitted from $\mathrm{C} 1$ to $\mathrm{C} 2$ by the concentration of free KaiA surrounding KaiC (red arrows). 

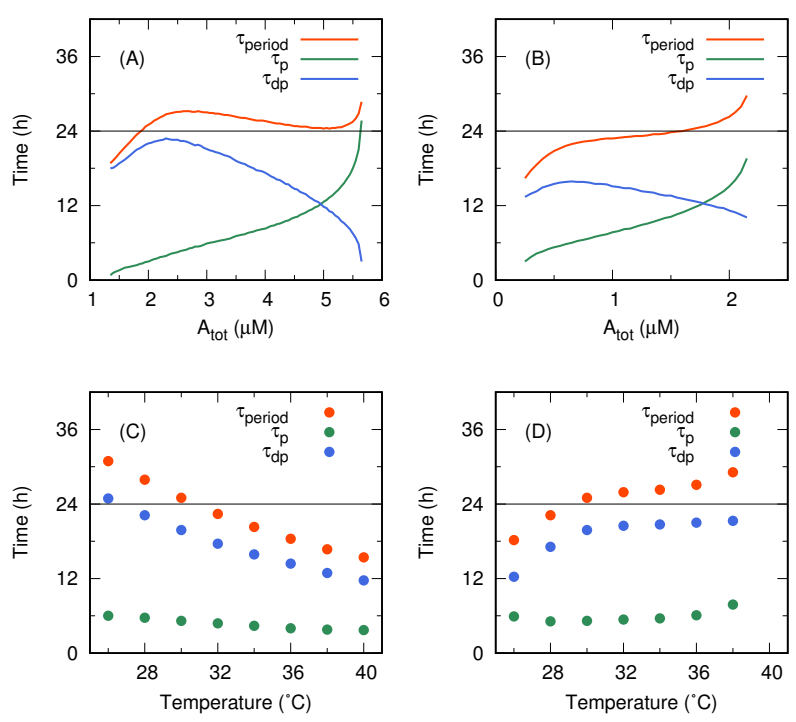

FIG. 5. Environmental parameter dependences of the periods in the present model. $\mathrm{A}_{\text {tot }}$ dependences of $\tau_{\text {period }}, \tau_{\mathrm{p}}$, and $\tau_{\mathrm{dp}}$ at $30{ }^{\circ} \mathrm{C}(\mathrm{A})$ when $k_{1 \mathrm{ex}}=5 \mathrm{~h}^{-1}$ and $(\mathrm{B}) k_{1 \mathrm{ex}}=30 \mathrm{~h}^{-1}$. Temperature dependences of $\tau_{\text {period }}, \tau_{\mathrm{p}}$, and $\tau_{\mathrm{dp}}(\mathrm{C})$ when the activation energy difference of $K_{2 \mathrm{~A}}$ is $0 \mathrm{kcal} / \mathrm{mol}$ and (D) is $-80 \mathrm{kcal} / \mathrm{mol}$. The pre-exponential factor of $K_{2 \mathrm{~A}}$ is determined so that $K_{2 \mathrm{~A}}$ coincides with its optimized value at $30^{\circ} \mathrm{C}$. 
TABLE I. Optimized parameters

\begin{tabular}{|c|c|c|c|}
\hline Parameter & Value at $30^{\circ} \mathrm{C}$ & $\begin{array}{r}\text { Activation energy or } \\
\text { activation energy difference } \\
(\mathrm{kcal} / \mathrm{mol})\end{array}$ & Constraint \\
\hline$\left[\mathrm{A}_{2}\right]_{\text {tot }}$ & $6.00 \times 10^{-1} \mu \mathrm{M}$ & 0 & Fixed \\
\hline$[\mathrm{B}]_{\text {tot }}$ & $3.50 \times 10^{0} \mu \mathrm{M}$ & 0 & Fixed \\
\hline$\left[\mathrm{C}_{6}\right]_{\text {tot }}$ & $5.83 \times 10^{-1} \mu \mathrm{M}$ & 0 & Fixed \\
\hline$k_{1 \mathrm{~h}}$ & $5.47 \times 10^{-1} \mathrm{~h}^{-1}$ & $2.36 \times 10^{0}$ & \\
\hline$k_{1 \mathrm{ex}}^{\mathrm{BIF}}$ & $1.28 \times 10^{1} \mathrm{~h}^{-1}$ & $7.68 \times 10^{-1}$ & \\
\hline$k_{1 \mathrm{ex}}^{\text {other }}$ & $0 \mathrm{~h}^{-1}$ & 0 & Fixed \\
\hline$k_{1 \mathrm{IC}, 0}$ & $5.92 \times 10^{-3} \mathrm{~h}^{-1}$ & $5.37 \times 10^{-1}$ & \\
\hline$\delta \mu_{1 \mathrm{ADP}}$ & $1.41 \times 10^{0}$ & $2.95 \times 10^{-2}$ & \\
\hline$k_{1 \mathrm{CI}}$ & $3.44 \times 10^{0} \mathrm{~h}^{-1}$ & $2.81 \times 10^{0}$ & \\
\hline$K_{1 \mathrm{~B}}$ & $1.0 \times 10^{-5} \mu \mathrm{M}$ & 0 & Fixed \\
\hline$K_{1 \mathrm{~A}}$ & $1.0 \times 10^{-5} \mu \mathrm{M}$ & 0 & Fixed \\
\hline$k_{2 \mathrm{~h}}$ & $2.49 \times 10^{-1} \mathrm{~h}^{-1}$ & $7.07 \times 10^{-1}$ & \\
\hline$k_{2 \mathrm{ex}}^{\mathrm{B}}$ & $0 \mathrm{~h}^{-1}$ & 0 & Fixed \\
\hline$k_{2 \mathrm{ex}}^{\mathrm{E}}$ & $6.77 \times 10^{0} \mathrm{~h}^{-1}$ & $9.86 \times 10^{-1}$ & \\
\hline$k_{\mathrm{S}+}^{\mathrm{R}}$ & $1.74 \times 10^{-1} \mathrm{~h}^{-1}$ & $3.00 \times 10^{1}$ & \\
\hline$k_{\mathrm{S}-}^{\mathrm{R}}$ & $1.69 \times 10^{-1} \mathrm{~h}^{-1}$ & $3.71 \times 10^{1}$ & \\
\hline$k_{\mathrm{S}+}^{\mathrm{F}}$ & $5.73 \times 10^{-1} \mathrm{~h}^{-1}$ & $5.05 \times 10^{-1}$ & \\
\hline$k_{\mathrm{S}-}^{\mathrm{F}}$ & $1.31 \times 10^{0} \mathrm{~h}^{-1}$ & $6.27 \times 10^{0}$ & Constrained by Eq. (19) \\
\hline$k_{\mathrm{T}+}^{\mathrm{R}}$ & $1.16 \times 10^{0} \mathrm{~h}^{-1}$ & $3.65 \times 10^{1}$ & \\
\hline$k_{\mathrm{T}-}^{\mathrm{R}}$ & $1.32 \times 10^{-1} \mathrm{~h}^{-1}$ & $4.04 \times 10^{1}$ & \\
\hline$k_{\mathrm{T}+}^{\mathrm{F}}$ & $2.10 \times 10^{-1} \mathrm{~h}^{-1}$ & $5.11 \times 10^{0}$ & \\
\hline$k_{\mathrm{T}-}^{\mathrm{F}}$ & $1.76 \times 10^{-1} \mathrm{~h}^{-1}$ & $1.72 \times 10^{1}$ & Constrained by Eq. (20) \\
\hline$K_{2 \mathrm{FR}, 0}^{\mathrm{B}}$ & $9.12 \times 10^{0}$ & $-5.67 \times 10^{0}$ & \\
\hline$\delta \mu_{2 \mathrm{pS}}$ & $1.08 \times 10^{0}$ & $-4.37 \times 10^{0}$ & \\
\hline$\delta \mu_{2 \mathrm{pT}}$ & $-7.92 \times 10^{-2}$ & $5.16 \times 10^{0}$ & \\
\hline$\delta \mu_{2 \mathrm{ADP}}$ & $-2.27 \times 10^{-1}$ & $3.02 \times 10^{0}$ & \\
\hline$K_{2 \mathrm{~EB}}^{R}$ & $\infty$ & 0 & Fixed \\
\hline$K_{2 \mathrm{~EB}}^{F}$ & $5.60 \times 10^{2}$ & $1.47 \times 10^{1}$ & \\
\hline$K_{2 \mathrm{~A}}$ & $1.14 \times 10^{-4} \mu \mathrm{M}$ & $-3.24 \times 10^{1}$ & \\
\hline
\end{tabular}

